\title{
Mitteilungen
}

Fähigkeitsausweis Homöopathie SVHA

Teilprüfung 1, deutsch

Diese kann nach Abschluss der Absolvierung der Grundlagenmodule abgelegt werden.

Ort: Sorell Hotel Arte Spreitenbach

Datum: Samstag, 21.9.2019

Anmeldefrist: 30.6 .2019

Weitere Informationen finden Sie unter www.svha.ch

Schweizerische Arbeitsgemeinschaft für Laparo- und Thorakoskopische Chirurgie (SALTC)

\section{Vorstand 2019}

Präsident

Prof. Dr. med. Dimitri Christoforidis, Lugano

\section{Past-Präsident}

Prof. Dr. med. Michel Adamina, Winterthur

\section{Sekretärin}

Dr. med. Diana Vetter, Zürich

\section{Vorstandsmitglieder}

PD Dr. med. Nicolas Buchs, Genf

Dr. med. Diego De Lorenzi, Grabs

Prof. Dr. med. Ulrich A. Dietz, Olten

PD Dr. med. Philipp Kirchhoff, Basel

Dr. med. Rebecca Kraus, Chur

Dr. med. Joël Lavanchy, Bern

Dr. med. Heidi Misteli, Uster

Dr. med. Marc-Olivier Sauvain, Lausanne

Dr. med. Emilie Uldry, Lausanne

\section{SALTC Sekretariat}

c/o Meister ConCept GmbH

Bahnhofstrasse 55

CH-5001 Aarau

Tel. 0628362090

Fax 0628362097

saltc[at]meister-concept.ch

www.saltc.ch

\section{Jean Wertheimer-Preis 2020}

Prof. Dr. med. Jean Wertheimer (1933-1999) war ein Pionier in der Alterspsychiatrie. Er wurde 1971 als der erste Lehrstuhlinhaber für Alterspsychiatrie in der Schweiz an die Universität Lausanne berufen, wo er eine weltweit anerkannte Alterspsychiatrie aufbaute. Später war er auch Präsident der Geriatric Psychiatry Section der WHO. Zu seinen Ehren hat die Schweizerische Gesellschaft für Alterspsychiatrie und Alterspsychotherapie einen Förderpreis für herausragende Arbeiten, wichtige innovative Projekte oder ethische Zielsetzungen im Sinne von Jean Wertheimer in der Alterspsychiatrie in der Schweiz geschaffen. Dieser wird alle zwei Jahre vergeben.

\section{Reglement für die Vergabe}

Ziel

Der Preis soll als Auszeichnung für hervorragende wissenschaftliche Originalarbeiten in klinischer alterspsychiatrischer Forschung, wichtige Projekte oder ethische Zielsetzungen vergeben werden. Die eingereichten Arbeiten sollen innovativ sein und müssen grosse klinische Relevanz aufweisen.

Der Jean Wertheimer-Preis wird ungeteilt an eine Person vergeben. Die Preissumme beträgt 5000 CHF. Der Preis wird anlässlich der Jahrestagung der Schweizerischen Gesellschaft für Alterspsychiatrie und -psychotherapie im Juni 2020 verliehen und muss vom Preisträger persönlich entgegengenommen werden.

Jury

Die Schweizerische Gesellschaft für Alterspsychiatrie und -psychotherapie hat eine Jury ernannt, die über die Vergabe des gestifteten Preises bis Ende Februar 2020 entscheiden wird.

Präsident: Prof. Dr. med. Urs P. Mosimann, Bern

Mitglieder: Dr. med. Michel Gaillard, Lausanne; Dr. med. Eva Krebs-Roubicek, Zürich; Dr. med. Nathalie Trächsel, Sion.
Die Jury ist in allen Entscheidungen frei und unabhängig. Die Entscheidungen sind nicht anfechtbar, der Rechtsweg ist ausgeschlossen. Arbeiten oder Bewerbungen, die nicht berücksichtigt wurden oder nicht berücksichtigt werden können, gehen ohne Begründung an den Absender zurück, ohne dass damit eine positive oder negative Bewertung getroffen worden ist. Die Vergabe kann sistiert werden, wenn die Qualität und Quantität der eingereichten Arbeiten ungenügend ist.

\section{Bewerbung}

Die eingereichten Arbeiten müssen vorwiegend in der Schweiz entstanden sein; der Arbeitsplatz des Initiators, Erstautors oder Senior-Autors soll bei der Einreichung in der Schweiz sein. Die Eingabe kann in deutscher, französischer, italienischer oder englischer Sprache erfolgen.

Folgende Unterlagen sind bei der Bewerbung beizulegen:

- Lebenslauf mit Publikationsliste

- Begleitschreiben, in welchem die Relevanz der beigelegten Arbeit dargelegt wird

- Eine Zusammenfassung der eingereichten Arbeit, max. eine A4-Seite

- Die Originalarbeit

- Die Arbeit darf nicht bereits einen anderen Wissenschaftspreis erhalten haben oder dafür ausgewählt sein. Gleichzeitige Einreichungen bei mehreren Preisausschreibungen müssen bei der Bewerbung offengelegt werden.

Die Bewerbung für den Jean WertheimerPreis 2020 ist schriftlich bis zum 30.11.2019 einzureichen bei:

Prof. Dr. med. Urs P. Mosimann

Direktor Medizin

Insel Gruppe AG

Inselspital, Universitätsspital Bern

Friedbühlstrasse 53

CH-3010 Bern

urspeter.mosimann[at]insel.ch 